\title{
Spinal Myoclonus after Subarachnoid Anesthesia with Bupivacaine
}

\author{
João Abrão ${ }^{1}$, Marcelo de Paula Bianco ${ }^{2}$, Waltuires Roma ${ }^{3}$, José Eduardo de Souza Krippa ${ }^{4}$, \\ Jaime Eduardo Hallak ${ }^{4}$
}

\begin{abstract}
Summary: Abrão J, Bianco MP, Roma W, Krippa JAS, Hallak JE - Spinal Myoclonus after Subarachnoid Anesthesia with Bupivacaine.
Background and objectives: It is presented in this case report a very rare complication after spinal anesthesia to provide subsidies to the management and therapeutic conduct.

Case report: This is a 63-year old African-Brazilian patient, ASA I, scheduled for transurethral resection of the prostate (TURP). He underwent subarachnoid anesthesia with bupivacaine $(15 \mathrm{mg})$ without adrenaline. Intercurrences were not observed during puncture, and the patient was positioned for surgery. Soon after positioning the patient, he complained of severe pain in the perineum region followed by involuntary tonic-clonic movements of the lower limbs. The patient was treated with a benzodiazepine to control the myoclonus without response. This episode was followed by significant agitation and the patient was intubated. He was maintained in controlled ventilation and transferred to the Intensive Care Unit. Despite all biochemical and imaging tests performed, an apparent cause was not detected. The medication was not changed and the same batch of anesthetic had been used in other patients that same day without intercurrences.
\end{abstract}

Conclusions: After ruling out all possible causes, the diagnosis of spinal myoclonus after spinal anesthesia with bupivacaine was made by exclusion.

Keywords: Anesthesia, Spinal; Myoclonus.

\section{INTRODUCTION}

Myoclonus is defined as the sudden development of involuntary contraction in a muscle group, single muscle, or part of a muscle. Spinal myoclonus has characteristics that differentiate it from other generalized myoclonus. In general, it is restricted to a few somatic regions and it is caused by diseases that involve the spinal cord ${ }^{1,2}$. The onset of myoclonus can be shortly after the stimulus of the spinal cord or after hours or days ${ }^{3,4}$. Any spinal cord stimulus, such as tumors, trauma, or degenerative processes can cause this phenomenon ${ }^{5}$. Spinal myoclonus can be induced by drugs injected during spinal anesthesia (anesthetics or opioids), radiologic tests (contrast), or placement of a subarachnoid catheter ${ }^{6}$. Spinal or epidural anesthesia-induced spinal myoclonus is extremely rare. The

Received from Faculdade de Medicina de Ribeirão Preto, Universidade de São Paulo (USP), Brazil.

1. Physician; Professor of the Disciplina de Anestesiologia, FMRP-USP

2. Urologist Santa Casa de Ituverava-SP,

3. Anesthesiologist Da Santa Casa de Ituverava- $S P$,

4. Professor of the Department of Neuroscience and Behaviour Sciences.

Submitted on December 17, 2010.

Approved on February 21, 2011

Correspondence to:

Dr. João Abrão

Rua Barão do Amazonas, 2.456/41

Jardim Sumaré

14025-110 - Ribeirão Preto, SP, Brazil

E-mail: joaoabrao@fmrp.usp.br objective of this paper was to report the occurrence of spinal myoclonus in a patient undergoing subarachnoid anesthesia with bupivacaine.

\section{CASE REPORT}

This is a 63-year old African-Brazilian patient, physical status ASA I, approximately $75 \mathrm{~kg}$, without history of seizures scheduled for transurethral resection of the prostate under spinal anesthesia. He was not premedicated. Monitoring consisted of electrocardiogram, non invasive blood pressure, and pulse oximetry. With the patient fully awake and on the sitting position spinal puncture was performed, successful on the first attempt, in the $\mathrm{L}_{3} / \mathrm{L}_{4}$ space with a $25 \mathrm{G}$ Quincke needle. After backflow of clear CSF, $0.5 \%$ bupivacaine and $7.5 \%$ glucose $3.0 \mathrm{~mL}(15 \mathrm{mg})$, were injected in the subarachnoid space. The injection was easily administered and the patient did not complain of pain. He was then placed in horizontal dorsal decubitus. When he first started feeling numbness on his legs, he was placed in the lithotomy position. At that moment, he also complained of an unbearable pain in the perineal region, which was followed by involuntary contractions in the muscles of the lower limbs. His heart rate increased to $120 \mathrm{bpm}$ and blood pressure to $170 / 90 \mathrm{mmHg}$. Diazepam $5 \mathrm{mg}$ IV was administered without results. The patient became agitated and difficult to control. The anesthesiologist decided to intubate him after the injection of $2.5 \%$ thiopental $(250 \mathrm{mg})$ and succinylcholine $(70 \mathrm{mg})$. To elucidate the diagnosis the CSF, 
which was clear, was collected and sent to the laboratory for analysis. Immediately, the anesthesiologist confirmed that the vial he used was indeed bupivacaine, and the batch of the local anesthetic was removed for analysis. The manufacturer of the anesthetic was informed of what had happened. The patient was transferred to the Intensive Care Unit sedated, intubated, and on assisted mechanical ventilation. In the ICU, $20 \mathrm{~mL}$ of blood were collected for biochemical and enzymatic analysis, and an MRI of the head and spinal column was requested. With the partial results of the tests, the diagnostic hypotheses were: extrapyramidal syndrome, myoclonus, or chemical myelitis. Immediate treatment with dexamethasone (4 mg four times a day), diazepam and phenytoin were instituted. Since all tests were normal, including the MRI, the patient was maintained on assisted ventilation and enteral nutrition until he could be weaned from the ventilator. The patient remained intubated for two days. On the third day he was completely awake and with voluntary movements of his lower limbs. He was then extubated, being discharged from the hospital on the following day without sequelae.

\section{DISCUSSION}

Spinal myoclonus is a reaction to a stimulus on a specific area of the spinal cord. The most striking characteristic is that the patient remains conscious. Contractions are repetitive, usually restricted to a muscle, or a group of muscles. They appear in varied time intervals, always corresponding to specific spinal innervation. Unlike other types of myoclonus, it is not affected by sleep, anesthesia, or coma. Contractions are rhythmic and may be synchronous in several muscles ${ }^{2}$. The pathophysiology of spinal myoclonus seems to be an abnormal hyperactivity of the local dorsal horn interneurons, with loss of inhibition of suprasegmental descending pathways ${ }^{7}$. This does not seem to explain the case presented here because contractions began shortly after the injection of local anesthetic. If it were related to the inhibitory function of the spinal cord, one would expect the contractions to appear in the regression of the spinal anesthesia. It is in this phase that the differential blockade is present. Most likely, an increased irritability of the $\alpha$-motor neurons was present, leading to the development of myoclonus caused by the anesthetic solution (glucose + local anesthetic). Alfa and Bamgbade ${ }^{8}$ described a case of spinal anesthesia for the surgical treatment of urocystocele in which the patient developed involuntary spastic movements of both lower limbs three hours after spinal anesthesia. Myoclonus was successfully treated with the intravenous injection of a total of $4 \mathrm{mg}$ of midazolam, which was titrated until it completely disappeared thirty minutes later. In the present study, since diazepam was able to control the contractions and the patient became progressively agitated, it was decided to treat him with deep sedation and secure an airway. This explains the ventilatory assistance. The hypothesis of changing the medication was ruled out after it was verified that the empty vial was of hyperbaric bupivacaine. The normal CSF examination ruled out the hypothesis of infection. That same day, other patients underwent spinal anesthesia with the same batch of local anesthetic and they did not develop similar manifestations, ruling out possible problems with the drug. In the ICU, the patient remained sedated and on mechanical ventilation; however, a few spastic contractions were observed. Since on the second day he did not show any signs of spinal irritability, the weaning process was started, the patient was extubated, and transferred to the regular ward on the third day, when he was also discharged from the hospital. The first case of myoclonus after spinal anesthesia in the literature was published by Fox et al. in $1979{ }^{9}$. They described a 57-year old woman who underwent transcutaneous ureterostomy under spinal anesthesia, in which the local anesthetic used was tetracaine $(14 \mathrm{mg})$ diluted in $10 \%$ glucose. Five hours after the surgery, she developed mild contractions on her right knee, followed by strong and irregular flexions/extensions of her thigh and knee, of short duration, and irregularly intermittent. Intravenous diazepam $2.5 \mathrm{mg}$ was used to control the muscle contractions. The patient had a full recovery and they did not find any disease. Latent spinal cord disease was attributed as the probable diagnosis. One should consider that, in this case, tetracaine was used, which is a more neurotoxic local anesthetic than bupivacaine. The patient in the present study returned to his rural work and, six months later, he was finally operated under general anesthesia without intercurrences. 